\title{
LA COMPRENSIÓN DE LA POBREZA EN UN GRUPO DE NIÑOS Y NIÑAS EN CONDICIÓN SOCIOECONÓMICA BAJA ${ }^{1}$
}

\section{THE UNDERSTANDING OF POVERTY IN A GROUP OF CHILDREN IN LOWER SOCIECONOMIC STATUS}

\author{
Randall Rodríguez Amor* \\ Adriana Salas Ruiz ${ }^{* *}$
}

RESUMEN

\begin{abstract}
En el artículo se exponen los resultados de una investigación sobre la comprensión de la pobreza (ideas y teorías) en un grupo de niños y niñas escolarizados de 7 a 12 años que viven en condición socioeconómica baja. Se demostró que manejan una conceptualización que va de lo concreto cotidiano a una noción más reflexiva y abstracta (de una concepción individualista de la pobreza hasta construir una definición más colectivista y relacional). Asimismo, sus teorías sobre el origen de la pobreza tienen características individualistas hasta tomar elementos de orden estructural social.
\end{abstract}

PALABRAS CLAVES: COSTA RICA * NIÑOS * NIÑAS * POBREZA * DESARROLLO DEL NIÑO * ASPECTOS PSICOLÓGICOS * PSICOLOGÍA

\section{ABSTRACT}

The article presents the results of a research about understanding of the poverty (ideas and theories) in a group of school boys and girls from 7 to 12 years living in lower socioeconomic status. It was shown that children handle a conceptualization that goes from a daily's concrete notion to a more reflexive and abstract one (from an individualistic conception of poverty to build a more collectivist and relational definition). Also, their theories about the origin of poverty have individualistic features till they also take elements of social structural order.

\section{KEY WORDS: COSTA RICA * CHILDREN * POVERTY * CHILD DEVELOPMENT * PSYCHOLOGICAL ASPECTS $*$ PSYCHOLOGY}

$1 \quad$ Este artículo surge de nuestra tesis de Licenciatura en Psicología "La comprensión infantil de la pobreza: ideas y teorías sobre la pobreza en un grupo de niños y niñas escolarizados de 7 a 12 años que viven en condición socioeconómica baja”, la cual se llevó a cabo con el apoyo de la beca de investigación del Instituto de Investigaciones Sociales de la Universidad de Costa Rica durante el año 2009.
* Instituto de Investigaciones Sociales de la Universidad de Costa Rica. RANDALL.RODRIGUEZ@ucr.ac.cr

** Cursa la Maestría en Psicología de la Educación de la Universidad de Costa Rica. adrisalasruiz@yahoo.es 


\section{INTRODUCCIÓN}

Este artículo brinda un aporte sobre las ideas, nociones o teorías en torno a la pobreza en la niñez en condición de desventaja económica. En este sentido, la investigación tiene una orientación hacia la psicología del desarrolo cognitivo desde una perspectiva que integra elementos contextuales mediante el uso del método de comparación constante (construcción de la teoría a partir de los datos generados con la población de estudio). Así, para llevar a cabo la investigación, fue central considerar la pobreza no sólo como un fenómeno de naturaleza meramente económica (carencias), sino un complejo estado de elementos sociales, culturales $y$ de conciencia individual, que se traducen en universos materiales y sobre todo simbólicos cognitivos (Morente y Barroso, 2003).

De este modo, ¿cómo entienden la pobreza un grupo de niños y niñas que viven en condición socioeconómica baja?, ¿cuáles son las teorías en términos de causas y consecuencias de la pobreza que tienen los mismos?, y ¿qué características asumen las ideas y teorías sobre la pobreza a lo largo de un tramo de desarrollo que va de los 7 a los 12 años en dicha población?, son las interrogantes que guiaron la investigación.

\section{ANTECEDENTES}

Una gran cantidad de trabajos han abordado las ideas o representaciones en la niñez sobre las instituciones económicas, sociales y políticas. Muchas de estas parten de las tempranas investigaciones de Piaget y Weil (1951), quienes estudiaron la formación intelectual y afectiva de la idea de patria en la niñez a inicios de los años cincuenta. En décadas posteriores, la teoría de Piaget ha influido en estudios sobre el desarrollo del juicio moral, la comprensión de las instituciones políticas, la estratificación y movilidad social, la autoridad escolar entre otros temas (Castorina, 2006).

Aquí se reseñan algunos hallazgos de estudios que se consideran pertinentes al tema, en este sentido, Faigenbaum (2000) identificó que los niños y niñas son agentes sociales que participan en diversas prácticas e interacciones comunicativas con sus pares y con los adultos. De este modo, elaboran ideas personales sobre fenómenos políticos, económicos, sociales e históricos. A su vez, dicha construcción está estrechamente asociada con creencias sociales que les preexisten y de las que se apropian en su relación con figuras adultas.

Por su parte, Amar, Abello, Denegri, Jiménez y Llanos (2006) encontraron cuatro niveles de desarrollo conceptual con respecto a las representaciones sociales de la pobreza y la desigualdad social, lo cual presenta una clara secuencia evolutiva.

En cuanto a la investigación costarricense, se pueden identificar importantes hallazgos sobre el desarrollo del pensamiento social-económico en la niñez. En este sentido, Dobles y González (1980) muestran que los niños y niñas consideran a los empresarios y profesionales como los grupos sociales con mayor prestigio y posibilidades en las esferas económica y social, en tanto que los campesinos son vistos como los más trabajadores. Asimismo, hay una preocupación de la población de estudio por formar parte de la clase baja, ya que esta condición es considerada como menos afortunados y con problemas sociales importantes. De este modo, la identificación de la pobreza en otras personas en situaciones más difíciles $y$ apremiantes, es un mecanismo que permite proteger la imagen que se tiene de sí mismo ya que las condiciones de carencia es un elemento clave en la conformación de la identidad personal (Garro, 2006).

Por su parte, el desarrollo de los conceptos e ideas en la niñez retoman elementos del discurso ideológico imperante en la sociedad, lo cual surge de la vivencia personal $y$ las experiencias comunitarias asociadas (Ayales y Flores, 1991). Asimismo, no sólo depende de la capacidad cognoscitiva sino también de todo un sistema de creencias que mantiene la idea de un mundo justo como regla universal (Gerchberg, 1994).

Así, en la niñez existe la capacidad de formular teorías sobre el mundo social en aspectos claves de la interacción como es la formación de la identidad étnica (Smith, 1995), o sobre el funcionamiento del subsistema económico (Campos, 2001), lo cual se rige por una lógica evolutiva. De este modo, el pensamiento se complejiza y tiene la capacidad de articular 
aspectos como intencionalidades, creencias, valores y emociones morales (Sánchez, 2007).

\section{METODOLOGÍA}

La estrategia metodológica que se consideró idónea para el estudio es de corte cualitativo ya que se buscó profundizar en la comprensión sobre las ideas que tienen los niños y las niñas del fenómeno de la pobreza. En este sentido, la investigación es un estudio cualitativo de tipo descriptivo, cuyos resultados se analizaron mediante el Método de Comparación Constante (Teoría Fundamentada).

De este modo, con un primer grupo de investigación, se utilizaron dos guías de entrevistas para la construcción y selección de un conjunto de tarjetas de dibujos sobre la temática de la pobreza.

Posteriormente, con un segundo grupo de niños y niñas, se profundizó en el fenómeno en cuestión mediante la utilización de un cuento con frases incompletas en conjunto con las tarjetas diseñadas.

\section{PRIMERA GUÍA DE ENTREVISTA}

La primera guía consta de siete preguntas divididas en dos apartados; el primer apartado con cuatro preguntas que recogen información personal (nombre, edad, residencia y género), $y$ el segundo apartado que indaga la definición y percepción sobre la pobreza según la perspectiva del niño o niña. Se recurrió al criterio experto para validar el instrumento.

\section{TARJETAS DE DIBUJO}

Las tarjetas de dibujo se construyeron a partir de la sistematización de las respuestas de la primera guía de preguntas. En este sentido, se pensaron las tarjetas como reactivos para generar las ideas y teorías sobre la pobreza.

En primera instancia se realizaron doce tarjetas de dibujo, se seleccionaron seis mediante criterio experto de las cuales finalmente quedaron tres seleccionadas por los niños y niñas.
Esto último se llevó a cabo mediante la aplicación de la segunda guía de preguntas la cual se construyó a partir de la primera guía por lo que no requirió validación.

\section{EL CUENTO}

Se construyó un cuento con frases incompletas titulado "Acerino el preguntón" el cual consiste en completar las preguntas del personaje principal durante la narración del mismo. La utilización del cuento se hizo en combinación con las tarjetas de dibujo como elementos ilustrativos y reactivos a la hora de hacer las preguntas. El diseño del cuento siguió una lógica lúdica para hacer más cómoda y flexible la metodología. Asimismo, cada sesión de trabajo inició con una actividad de animación para generar confianza y soltura, posteriormente, se pasó a la producción gráfica donde se le pidió al niño o niña realizar un dibujo sobre su concepto de pobreza para finalmente terminar con el cuento en mención.

\section{LA MUESTRA DE ESTUDIO}

La selección de los sujetos correspondió a criterios de homogenización tales como: condición socioeconómica baja, proporcionalidad en cuanto al género, edad y número de sujetos por cada nivel escolar.

Se trabajó en la Escuela Finca La Caja de la Carpio, esta comunidad se ubica en el distrito de la Uruca del Cantón Central de San José (Capital de Costa Rica), dicha comunidad cuenta con una concentración importante de pobreza urbana y exclusión social (Sandoval, 2005).

Inicialmente se diseñó una muestra de veinticuatro sujetos: un niño $y$ una niña por cada nivel académico (de primer a sexto grado escolar) en dos grupos de investigación (doce para cada grupo).

Al final del estudio participaron en total 42 individuos, 9 niños y 10 niñas para la primera parte de la investigación; y 11 niños y 12 niñas para la segunda parte, se decidió aumentar la cantidad de personas dado el elevado ausentismo en la institución educativa. 
RESULTADOS

ELEMENTOS DE LA PRIMERA GUÍA DE PREGUNTAS

Como fase previa para trabajar las ideas en torno a la pobreza se planteó la construcción de las tarjetas de dibujos como instrumento de recolección de información. En este sentido, las respuestas del primer grupo fueron las representaciones e ideas iniciales que dan cuenta de este aspecto (cuadro 1), lo cual se amplió posteriormente con el grupo final de investigación.

CUADRO 1

RESPUESTAS DE LA PRIMERA GUÍA, SEGÚN GÉNERO

\begin{tabular}{lcc}
\hline PREGUNTAS Y RESPUESTAS & \multicolumn{2}{c}{ FRECUENCIA DE RESPUESTAS } \\
\hline ¿PARA VOS QUÉ ES SER POBRE? & NIÑAS & NIÑOS \\
\hline Sin dinero & 3 & 3 \\
No pueden comprar comida, buena ropa o cosas materiales & 7 & 6 \\
No tienen donde vivir o viven en la calle & 2 & 3 \\
No pueden acceder a cosas como diversión o estudio & 2 & NIÑOS \\
\hline ¿ĆMO CREES VOS QUE SON LAS PERSONAS POBRES? & NIÑAS & 2 \\
\hline Son buenas personas & 0 & 3 \\
Viven en precarios, las casas no tienen nada & 2 & 6 \\
Su aspecto es muy deteriorado (flacos, tristes y ropa en mal estado) & 6 & 1 \\
Indigencia & 3 & NIÑOS \\
\hline CóMO CREES VOS QUE VIVEN LAS PERSONAS POBRES? & NIÑAS & 7 \\
\hline Viven en la calle, son indigentes & 5 & 4 \\
\hline
\end{tabular}

Como se puede apreciar en las respuestas, la pobreza se enlaza a elementos concretos de orden material principalmente, como es la falta de ropa, comida o vivir en condiciones precarizadas, elementos que se resaltaron en el diseño de las tarjetas, a continuación se presentan las tarjetas 1 y 2 seleccionadas por los niños: 
TARJETA 1

GÉNERO MASCULINO

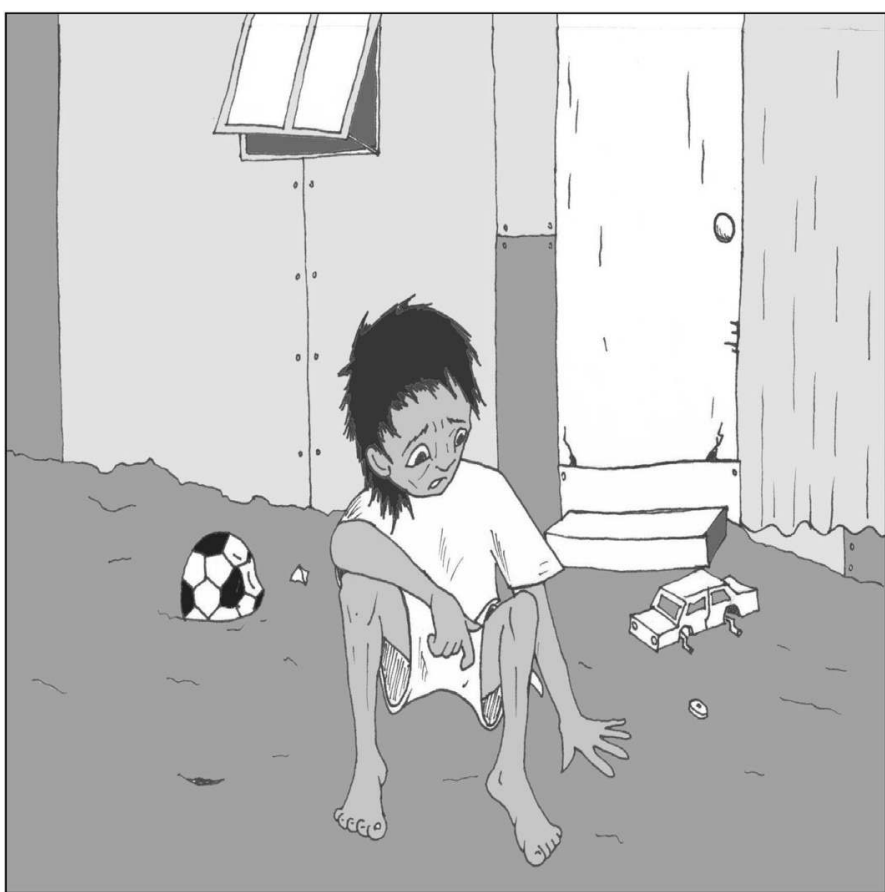

Dibujante: Jorge Solano Cantillo.

TARJETA 2

GÉNERO MASCULINO

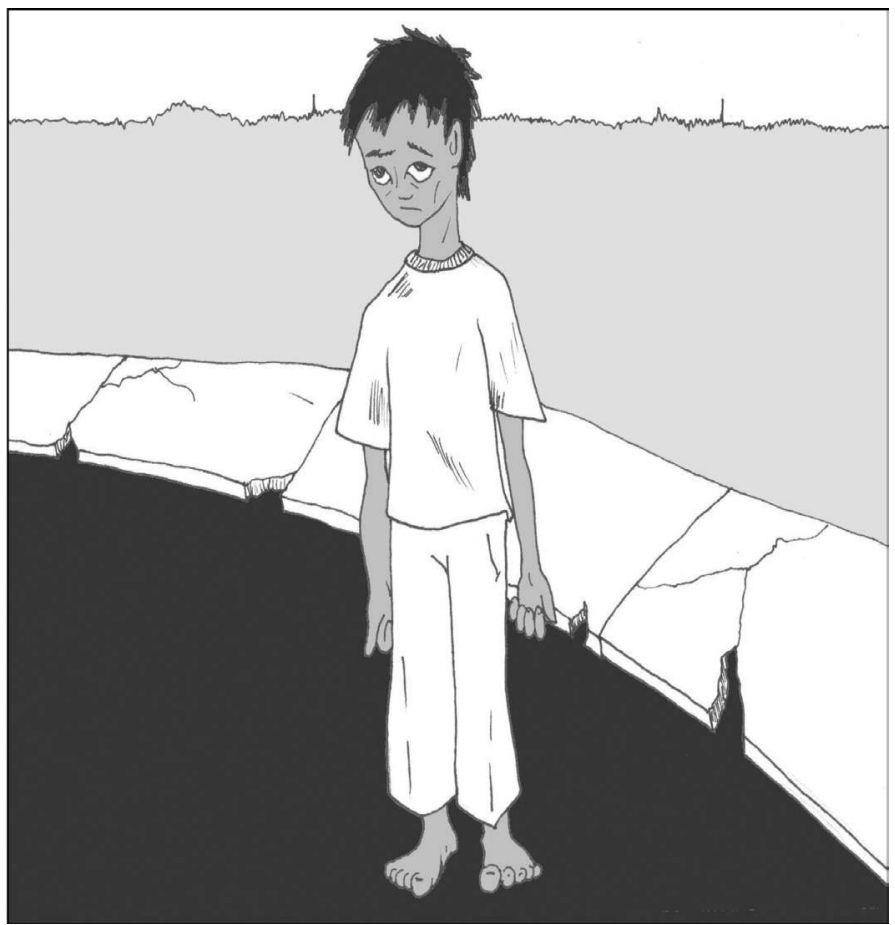

Dibujante: Jorge Solano Cantillo.

Rev. Ciencias Sociales Universidad de Costa Rica, 126-127: 11-24 / 2009-2010 (IV-I). (ISSN: 0482-5276) 


\section{CONCEPTUALIZACIÓN DE LA POBREZA}

A partir de los elementos mencionados por los niños y niñas del segundo grupo de investigación fue posible identificar los componentes de la definición, los cuales dan cuenta de los matices conceptuales que se tienen en torno a la representación cognitiva de la pobreza que se presentan a continuación:

\section{ESTRATIFICACIÓN}

A pesar de que se describe un grupo bastante homogéneo de personas, se pueden establecer dos niveles. El primero, se refiere a las personas que necesitan de la caridad pero tienen vivienda (no es del todo pobreza extrema). El segundo nivel, identifica la pobreza con condiciones extremas de carencia material (mendicidad con indigencia).

En el caso de los niños y niñas, se pueden identificar dos tipos de estratificación. El tipo uno, que sólo contempla el primer nivel de la pobreza, $y$ un segundo tipo, que considera los dos niveles.

\section{TIPOS DE CARENCIA}

La definición de la pobreza se relaciona con las carencias, las cuales pueden ser de índole material (falta de dinero, ropa, comida, vivienda $y$ automóvil), y las que contemplan otros derechos $y$ aspectos emocionales junto a las carencias materiales (falta de educación, seguridad $y$ control sobre la propia vida).

\section{TIPOS DE EXPERIENCIA}

En las definiciones de la pobreza se pueden identificar dos tipos. La primera que relaciona la pobreza con una experiencia individual (se menciona a una sola persona que por lo general es adulta), $y$ la segunda contempla una experiencia colectiva de la misma (se mencionan personas adultas, niños o niñas, asimismo, se incluye a la familia o la comunidad).

\section{MODO DE IDENTIFICACIÓN}

Se refiere a los elementos que sirven para identificar a las personas en condición de pobreza. En este sentido, está el modo que se basa en la apariencia de las personas (suciedad, ropa deteriorada, delgadez como elementos centrales), y el modo que contempla, además de los mencionados, otros elementos de estatus como es el automóvil y las condiciones de la vivienda.

En todos los casos se tiene claro la función del dinero como instrumento de intercambio para obtener bienes y servicios en la interacción social.

En el cuadro 2 se muestran la ubicación de la población de estudio con respecto a los componentes identificados. 


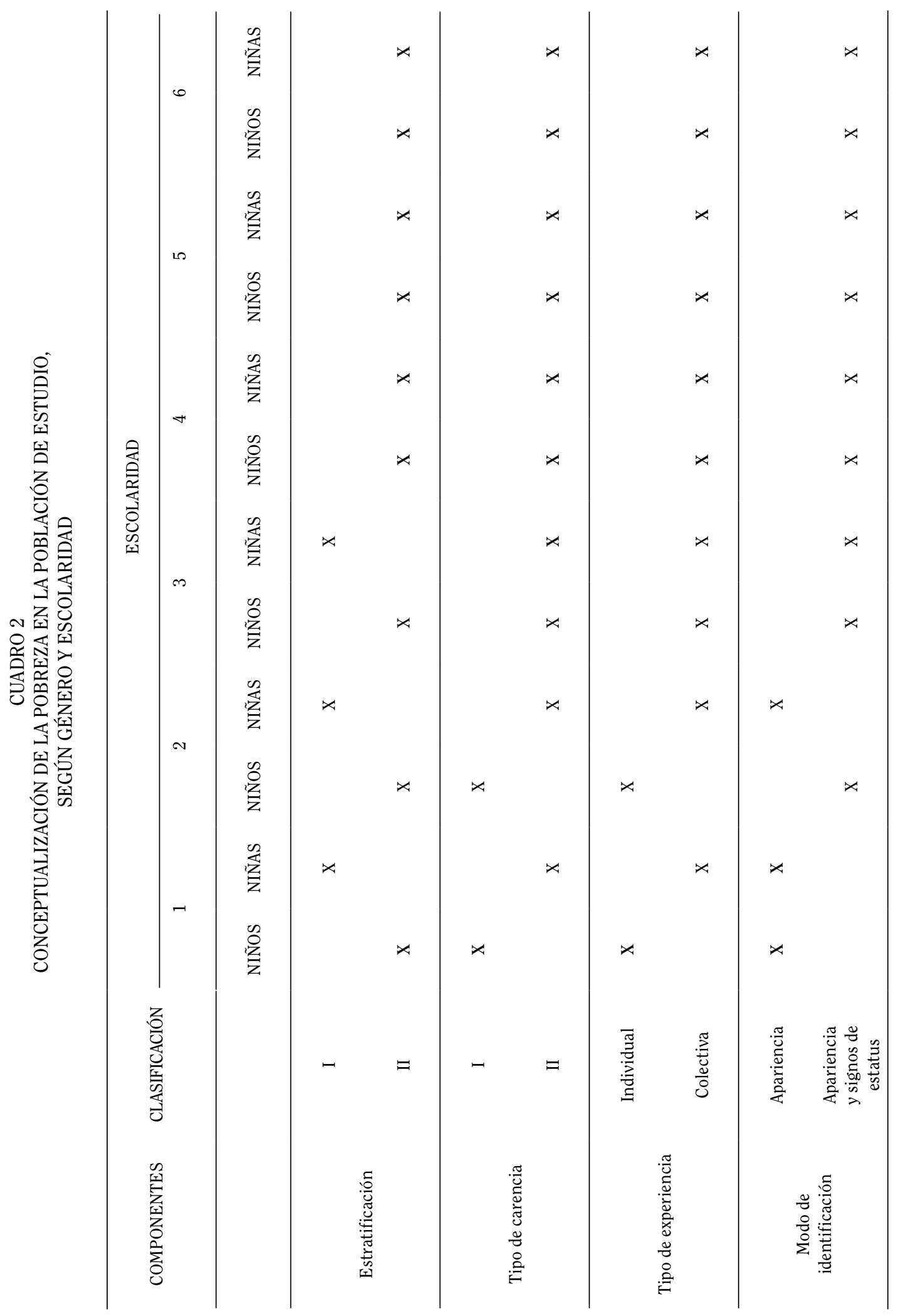

Rev. Ciencias Sociales Universidad de Costa Rica, 126-127: 11-24 / 2009-2010 (IV-I). (ISSN: 0482-5276) 
ORIGEN DE LA POBREZA

El origen de la pobreza constituye una explicación del funcionamiento de la sociedad en cuanto al acceso a los recursos, en los niños y niñas se pudieron identificar dos tendencias: una más simple $y$ directa, a la que se denomina Pensamiento de Tipo A (PTA) y otra de carácter más elaborado, llamada Pensamiento de Tipo B (РTB).
El pensamiento de Tipo A se observó con mayor frecuencia entre los niños y niñas de primer ciclo (primero, segundo y tercer grado), en tanto que el pensamiento de Tipo $B$ está más presente en los niños y niñas del segundo ciclo (cuarto, quinto y sexto grado).

Las principales características de cada tipo se pueden apreciar en el siguiente cuadro:

CUADRO 3

CARACTERÍSTICAS DE CADA TIPO DE PENSAMIENTO

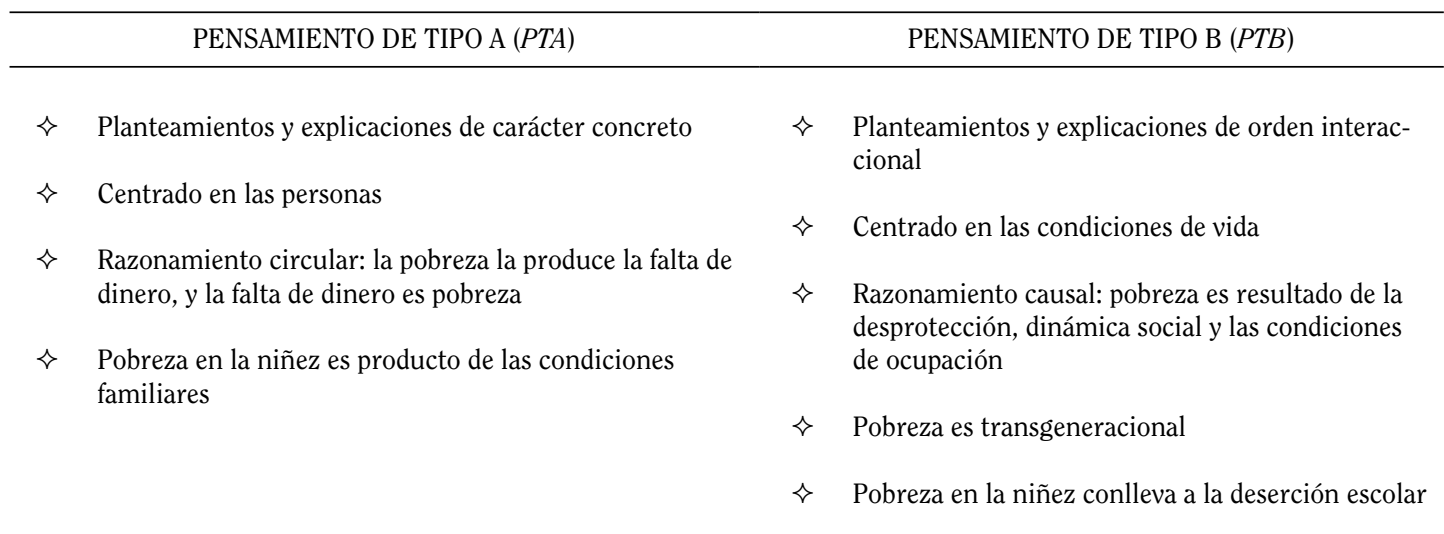

PENSAMIENTO DE TIPO A

El pensamiento de Tipo A, se caracteriza por estar basados en planteamientos $y$ explicaciones de carácter concreto, centrados en las personas $y$ de razonamiento circular en ocasiones. Es decir, para los participantes en PTA la pobreza reúne características concretas como es la ropa o la mendicidad y su origen radica en la falta de dinero y a la vez la falta de dinero es producto de la pobreza. De igual manera tienden a dar explicaciones individualistas, siendo que los y las niñas en este nivel aluden a que la pobreza se origina y sostiene por el comportamiento de las personas.

Así pues, para los niños y niñas ubicados en el PTA las personas en condición de pobreza están ahí porque no trabajan y les falta dinero. En este sentido, el razonamiento es bastante claro, el dinero se obtiene trabajando, si no se trabaja por ende se carece de dinero para cubrir las necesidades de alimento, vestido y casa.
PENSAMIENTO DE TIPO B

En el caso de los niños y niñas de segundo ciclo de Educación General Básica se observó que en general sus planteamientos y explicaciones en torno al origen de la pobreza se caracterizan por ser predominantemente de orden interaccional. Es decir, para ellos la pobreza es resultado del abandono o el desamparo, así como de la falta de voluntad por parte de las personas o instancias percibidas como capaces de solucionar esta condición: los ricos y el Estado, lo que supone una responsabilidad más colectiva.

Ya la pobreza no es enteramente una circunstancia individual, es decir las explicaciones dejan de centrarse en las actitudes de "la persona pobre" para considerar las circunstancias que existen en el entorno y que hacen que la pobreza permanezca (descentramiento cognitivo).

En términos generales para los niños y niñas ubicados en PTB las causas de la pobreza 
en su manifestación más concreta están en las condiciones de vida como es la falta de trabajo y problemas sociales como el abandono y la indigencia.

\section{LA DESIGUALDAD}

La noción de desigualdad como aspecto lógico e inseparable de la pobreza, es bastante simple y homogénea a lo largo de los seis niveles escolares, sin embargo, existen algunas diferencias importantes entre niños y niñas, lo cual se sintetiza en el cuadro 4.

Para la población de estudio la desigualdad significa asimetría en la obtención y tenencia de recursos, representada primordialmente por el dinero, aunado, en algunos casos, al acceso a determinados servicios o derechos lo que

CUADRO 4

CARACTERÍSTICAS DE LA DESIGUALDAD, SEGÚN GÉNERO

\section{DESIGUALDAD}

Características compartidas a nivel discursivo:

$\diamond \quad$ Pobreza está enlazada a los recursos (dinero)

$\diamond \quad$ Derechos a los que no se acceden

\begin{tabular}{llll}
\hline & \multicolumn{1}{c}{ LAS MUJERES ENFATIZAN } & & \multicolumn{1}{c}{ LOS HOMBRES ENFATIZAN } \\
\hline & Dos grupos de personas antagónicos (ricos y pobres) & $\diamond$ & Calidad de vida (condiciones) \\
& donde unos tienen lo que los otros no tienen & $\diamond$ & Diferentes niveles de pobreza \\
$\diamond \quad$ Situaciones en que se violentan constantemente los & $\diamond$ & La riqueza se mira como ideal deseable \\
& derechos fundamentales & $\diamond$ & La casa como signo de estatus y diferenciación \\
& Relatos en torno a las amenazas a la seguridad física con & & social \\
& todo lo que ello implica & $\diamond$ & Distribución desigual de recursos a nivel social \\
\hline
\end{tabular}

supone definiciones más complejas. Asimismo, se identifican dos polos opuestos claramente identificables en la representación de la desigualdad, es decir los ricos y los pobres. En este sentido, la riqueza se mira como ideal deseable para tener una vida lejos de la tristeza y desesperación, lo cual se relaciona claramente con las condiciones de la vivienda como signo de ubicación social o estatus ("las personas ricas viven en buenas casas").

La riqueza representa una vida agradable y cómoda, sin embargo, los niños del sexto grado hacen una lectura particularmente interesante sobre la desigualdad, ellos mencionan que lo malo de la pobreza es observar los recursos que otras personas tienen y no aprecian 0 desperdician, en tanto hacen un uso incorrecto de los mismos, lo que implica una atribución de falta de solidaridad en este estrato.

Al tratar el tema de la desigualdad frecuentemente apareció la representación del mundo ocupacional cuyas características se muestran en el cuadro 5.

Aunque en términos generales las percepciones sobre el mundo ocupacional fueron bastante homogéneas, en las mujeres el espacio socio laboral es un poco más variado y los hombres tendieron a establecer relaciones directas entre esta categoría y la falta de oportunidades. Pese a ello, en ambos grupos el perfil ocupacional es muy limitado aunque se tiene plena noción de que las personas obtienen recursos mediante un intercambio de fuerza de trabajo por dinero; sin embargo, no se hace diferenciación alguna sobre el estatus o posiciones que se ocupan en el mercado laboral. Por su parte, los trabajos que se mencionan son de baja calificación, debido posiblemente al ambiente socializador inmediato que concentra mayormente este tipo de labores. En este sentido, no se tiene claro el papel de las profesiones para superar la pobreza lo que hace que cualquier ocupa- 
CUADRO 5

CARACTERÍSTICAS DEL MUNDO OCUPACIONAL, SEGÚN GÉNERO

\section{REPRESENTACIÓN DEL MUNDO OCUPACIONAL}

Características compartidas a nivel discursivo:

$\diamond \quad$ Perfil ocupacional limitado

$\diamond \quad$ Trabajos de baja calificación son considerados adecuados

$\diamond \quad$ No se tiene claro el papel de las profesiones

$\diamond \quad$ Las condiciones de desocupación son generadoras de pobreza

\begin{tabular}{ll}
\hline LAS MUJERES ENFATIZAN & LOS HOMBRES ENFATIZAN \\
\hline$\diamond \quad$ Espacio socio laboral más variado & $\diamond$ Relación entre espacio socio laboral y la falta de oportunidades \\
& $\diamond$ Relación entre desigualdad en el ingreso y una vida mejor \\
\hline
\end{tabular}

ción sirva de igual manera. Esto último se relaciona con las ideas o nociones sobre la superación o solución a la pobreza, aspecto que se pudo observar en dos niveles de pen- samiento, el Nivel de Pensamiento General (NPG) y el Nivel de Pensamiento Ampliado (NPA), cuyas características se muestran a continuación:

\section{CUADRO 6}

CARACTERÍSTICAS DE LOS MODELOS DE SOLUCIÓN DE LA POBREZA, SEGÚN LOS NIÑOS Y NIÑAS

\begin{tabular}{cll}
\hline NIVEL DE PENSAMIENTO GENERAL $(N P G)$ & NIVEL DE PENSAMIENTO AMPLIADO $(N P A)$ \\
\hline $\begin{array}{l}\text { Propuesta unidireccional de solución centrada en } \\
\text { la persona pobre }\end{array}$ & $\diamond$ & $\begin{array}{l}\text { Propuestas multidireccionales que implican un } \\
\text { orden interaccional }\end{array}$ \\
$\diamond \quad$ Resolver las carencias materiales de la persona & $\diamond$ & $\begin{array}{l}\text { Se establece una relación entre trabajo y estudio } \\
\text { Vías: trabajo y/o la caridad }\end{array}$ \\
& $\diamond \begin{array}{l}\text { Surge la figura del Gobierno como encargado de } \\
\text { resolver la pobreza }\end{array}$ \\
\hline
\end{tabular}

El nivel general se destaca por dos soluciones a la pobreza: el trabajo y la caridad. Este nivel atraviesa todos los niveles educativos.

Por otra parte, el nivel ampliado comparte características propias del nivel general pero además incluye elementos que implican un orden interaccional o que necesariamente involucra a otras personas en la solución de la pobreza, se presenta principalmente en los niños y niñas de segundo ciclo quienes en sus respuestas intercalan elaboraciones que dan cuenta de una visión más amplia respecto a la temática en particular.
LA POBREZA EN LA VIDA DE LAS PERSONAS DESDE LA PERSPECTIVA DE LOS NIÑOS Y NIÑAS

En los niños y niñas la pobreza como concepto se resume como la falta de recursos económicos o dinero para satisfacer las necesidades básicas y de manera más compleja y comprensiva el derecho a la educación, diversión y consumo no asociado a la comida (calidad de vida). En este sentido, las carencias materiales producen infelicidad, condición fuertemente asociada a la pobreza y resume una visión trágica de las circunstancias presentes en todos los niveles escolares y en ambos géneros. 
En el caso de los niños, hasta el tercer grado, la pobreza se ve como una vivencia lejana sin relación con su vida, circunstancias o familia, no se asume como condición propia, se mira hacia abajo de la escala social para identificarla ("personas sin casa y/o que piden en la calle").

La relación pobreza y falta de calidad de vida (disfrute de derechos) se establece en los niños del cuarto grado en adelante, donde la definición se vuelve un poco más integral y compleja, en este sentido, es una imposibilidad real para acceder a los derechos que son percibidos como exclusivos de las personas con recursos (no pobres).

Los niños del cuarto grado hacen una asociación clara entre la desigualdad de recursos y la tristeza. Asimismo, la pobreza se vuelve molesta ya que establece una comparación con aquellos que tienen recursos para acceder a sus derechos. Dichos derechos (calidad de vida, diversión, comodidad) son percibidos como casi inalcanzables por las personas en pobreza a menos que se recurra a la solidaridad individual o colectiva.

Un aspecto central de la representación de los derechos es que se valoran como privilegios, suerte o producto de la caridad.

El ángulo negativo de la pobreza, es decir, su asociación con la carencia, el abandono y la tristeza, es permanente en el discurso de los niños y se vuelve reiterativo en cuarto, quinto y sexto grado donde se enfatiza su faceta depresiva (es un sufrimiento incapacitante).

De igual modo, las niñas asocian la pobreza con la tristeza en todos los grados. Asimismo, en quinto se contemplan otros estados de ánimo como es la soledad, el desánimo y la sensación de debilidad física y psicológica. Las niñas contemplan más experiencias en torno a la pobreza como es la posibilidad de humillación y mal trato familiar o social, aunado a la imposibilidad de jugar lo que afecta el disfrute en la niñez.

Las niñas de sexto grado perciben la pobreza como una condición de vulnerabilidad extrema lo que significa dependencia total hacia otras personas o la ayuda estatal. El estado de ánimo varía según las condiciones o circunstancias externas lo que hace de la tristeza una condición variable.
Las consecuencias de la pobreza para todas las edades $y$ en ambos géneros se relacionan principalmente con las condiciones de vida y consumo de las personas, dado que la pobreza se identifica con la mendicidad y la indigencia, las consecuencias son precisamente vivir de este modo, aunado a comunidades con insuficientes servicios, viviendas en malas condiciones o en riesgo importante de sufrir destrucción ante fenómenos naturales.

Desde un punto de vista individual se percibe como un estado de mayor exposición al maltrato físico y psicológico, las niñas insisten sobre los peligros a la integridad personal y al riesgo de sufrir violaciones o abusos. En este sentido, se es más vulnerable al peligro y a las circunstancias externas lo que implica una baja sensación de seguridad personal y comunitaria.

Socialmente la pobreza es percibida como productora de importantes problemas ya que induce al crimen como el robo, drogadicción y la violencia familiar $y$ social.

A pesar de que la pobreza es vista como algo trágico y desesperanzador, es posible identificar vivencias que atenúan o contrarresten dichos efectos. En este sentido, para los niños de segundo grado la unión familiar es fundamental $y$ deseable, aspecto que se aprecia en las niñas en general. Asimismo, se puede ser una persona honesta $y$ buena para los niños de tercer grado a pesar de las circunstancias.

Por su parte, la pobreza es percibida como una condición que moviliza la solidaridad individual o colectiva, elemento que se resalta como algo éticamente correcto en los niños y niñas. Lo anterior significa que la desesperanza no involucra todos los aspectos de la vida humana $y$ que existe espacio para otras vivencias a pesar de las carencias.

\section{CONCLUSIONES}

Trabajar en un tramo o rango de edad que comprende una etapa de desarrollo cognitivo donde el pensamiento abstracto aún no está presente supuso un serio obstáculo para una propuesta que se centró justamente en procesos de abstracción complejos como son la elaboración de teorías. Sin embargo, encontramos que 
los niños y niñas crean un mundo de significados e inclusive explicaciones de los fenómenos que obedecen a su vivencia concreta inmediata y cotidiana, marco general para la construcción posterior de la abstracción misma, aspecto que también ha sido encontrado en otros contextos y estudios.

El punto de partida que sostuvo toda la investigación fue considerar que los niños $y$ niñas elaboran un sentido sobre los fenómenos que se producen en la sociedad en tanto participan y son parte de ella, aspecto que se va complejizando a lo largo del desarrollo. En este sentido, la perspectiva metodológica que se desarrolló a lo largo del trabajo brinda algunos indicios de que la comprensión del mundo social en la niñez sigue un camino progresivo y se complejiza según la edad.

Los niños y niñas manejan una conceptualización que va de lo concreto cotidiano a algo un poco más reflexivo y abstracto (de una concepción individualista de la pobreza hasta construir una definición más colectivista y relacional). Esto último guarda características propias en cada género, aspecto al que no se le ha prestado la suficiente atención en la literatura científica consultada.

El marco interpretativo para definir la pobreza o las nociones de la desigualdad son de naturaleza económica en los tres primeros niveles escolares y fundamentalmente en los niños, lo que implica una noción de línea de pobreza a partir de los recursos económicos. De este modo, en ambos géneros y en todos los niveles se insiste sobre esta representación, sin embargo, conforme se avanza en los últimos niveles escolares se toman en cuenta elementos de estatus y acceso a otros bienes simbólicos y culturales (derechos como educación, salud, vivienda en buenas condiciones, calidad de vida $y$ posibilidades de disfrute).

En la población de estudio el principal signo de identificación y que sirve también para articular una representación de niveles o estratificación social dentro de la pobreza corresponde a la vivienda, sus condiciones y tenencia, implicando una noción que aunque toma en cuenta algunas diferencias sociales y estratificación es bastante simple y concreta. Este dato corresponde a un nivel de desarrollo de las representaciones de pobreza que estaría entre un primer y segundo nivel según el trabajo de Amar, et ál. (2001). Al parecer las condiciones contextuales permiten desarrollar nociones bastante consistentes a su edad a pesar de las limitaciones de estímulo escolar y ambiental que experimentan dichos niños y niñas.

En ellos y ellas el elemento que articula la representación de la carencia material es el dinero lo que indica una comprensión de su función en la sociedad, al menos en su función de intercambio, lo que confirma su importancia para el desarrollo de la comprensión del mundo económico (Campos, 2001).

Por otra parte, se replicaron los resultados de otras investigaciones donde la relación pobreza y carencias de recursos constituye un elemento central en la construcción conceptual de las personas (Nayaran, 2000). Asimismo, la pobreza significa una profunda vivencia de tristeza, desesperación, incapacidad, maltrato y una condición importante de problemas sociales e individuales, aunque es posible identificar vivencias positivas a pesar de las carencias materiales como es la unión familiar y el juego (no es del todo un destino trágico). Esto último tiene implicaciones para el abordaje metodológico con poblaciones empobrecidas o marginalizadas. La construcción de alternativas políticas para el combate de la pobreza y la exclusión requiere una visión esperanzadora (es posible tener calidad de vida), aspecto que se considera fundamental incentivar desde la infancia en la socialización familiar y comunitaria, aunado a un esfuerzo educativo que tome en cuenta los elementos y dinámicas estructurales para la comprensión de la exclusión, lo cual es posible dados los elementos reseñados por los niños y niñas. Así, la falta de trabajo como causa de pobreza implica una noción sobre su origen social y no como algo natural o dado. En este sentido, el perfil ocupacional es muy limitado aunque se tiene cierta representación sobre las diferencias en las remuneraciones. Esto resulta en una definición funcionalista de las posiciones en la sociedad, en tanto los niños y niñas asumen que la pobreza es resultado del esfuerzo individual, existiendo una lógica de 
premios $y$ desventajas asociadas a dicho esfuerzo (meritocracia), a pesar de que se consideran otros factores para su superación (ayuda estatal o colectiva - caridad-y empleo). Esto último implica que los niños y niñas consideran algunos elementos básicos de carácter socio-estructural para explicar la pobreza y la desigualdad, por lo que es necesario producir oportunidades de capacitación y trabajo para superar dichas condiciones desde su perspectiva.

A pesar del importante papel que se le asigna al trabajo para superar la pobreza, no se encontró de manera consistente una clasificación o delimitación de estatus a partir de las profesiones, aspecto que contrapone a los hallazgos de Dobles y González (1980) donde los niños y niñas pudieron establecer diferencias acorde a la profesión u ocupación de las personas. En este sentido, se considera que el entorno sociolaboral inmediato donde las ocupaciones de las personas son pocas $y$ de baja calificación, genera dicho fenómeno.

Con algunas diferencias mínimas, la infelicidad y la pobreza son aspectos inseparables en todos los discursos sin distingo de edad o género. Lo que la hace una vivencia con profundas implicaciones en la subjetividad de los niños y niñas, aspecto registrado también en la literatura consultada.

En relación con lo anterior, existe una clara tendencia a identificar la pobreza en otros contextos o personas abajo de la escala social donde se está ubicado, de este modo, se describe en otras personas $y$ situaciones, como una manera de mantenerla lejos, ya que representa una situación de derrota, humillación, vulnerabilidad y debilidad. Esto confirma la existencia del fenómeno de la comparación social descendente, cuya función central es proteger la autoestima de los niños y niñas cuando se ven expuestos a situaciones en las cuales se sienten vulnerables (Garro, 2006).

\section{BIBLIOGRAFÍA}

Amar, J.; Abello, R.; Denegri, M.; Jiménez, G. y Llanos, M. La construcción de representaciones sociales de la pobreza y desigualdad social en los niños de la región Caribe colombiana. Colombia: Red de Investigación y Desarrollo, 2006.

Ayales, I. y Flores, D. "El concepto de paz en niños de 7 a 11 años de una comunidad rural, Jiménez de Pococí". [Tesis de Licenciatura en Psicología]. Universidad de Costa Rica, 1991.

Barroso, I. y Morente, F. "La precariedad familiar ante la pobreza de la infancia. Una aproximación sociológica”. Potuaria 3. Universidad de Huelva, 2003: 67-88. En: <http://dialnet.unirioja.es/servlet/ articulo? codigo $=808140$ yorden $=89622$ yinfo=link $>$. [Consultado 30 de agosto 2008].

Bogdan, R. y Taylor, S.J. Introducción a los métodos cualitativos de investigación. Barcelona: PAIDós, 1987.

Campos, D. El mundo infantil y el mundo económico. El desarrollo de las teorías infantiles sobre la economía. San José, Costa Rica: Editorial de la Universidad de Costa Rica, 2001.

Castorina, J. "La investigación psicológica de los conocimientos sociales. Los desafíos a la tradición constructivista". En: Castorina, J (coordinador). Construcción conceptual y representaciones sociales. El conocimiento de la sociedad. Buenos Aires. Gedisa, 2006: 20-44.

Corbin, J. y Strauss, A. Bases de la investigación cualitativa: técnicas y procedimientos para desarrollar la teoría fundamentada. Colombia: Universidad de Antioquia, 2002.

Corugat, F. y Emiliani, F. El mundo social de los niños. México: Grijalbo, 1991.

Davidziuk, A.; Delamónica, E. y Minujin, A. "Pobreza infantil: conceptos, medición y recomendaciones de políticas públicas". 
Cuaderno de Ciencias Sociales 140. FLACSO, 2006.

Dobles, I. y González, M. Desarrollo de esquemas conceptuales: estudio sobre la "forma de pensar" de niños escolares. San José, Costa Rica: Universidad de Costa Rica, 1980.

Faigenbaum, G. "Los criterios de valor económico en el niño". Castorina, J. y Lenzi, A. (compiladores). La formación de los conocimientos sociales en los niños. Investigaciones psicológicas y perspectivas educativas. Buenos Aires. Gedisa, 2000: 107-134.

Feldman, R. Desarrollo psicológico a través de la vida (4. ${ }^{\text {a }}$ edición). Massachusetts: Universidad de Massachusetts, 2006.

Garro, L. "Estigmas, miedos e imaginarios de futuro. La construcción de identidades juveniles en un contexto de pobreza". [Tesis de Doctorado en Estudios Científicos Sociales]. Tlaquepaoue, Jalisco, México. Instituto Tecnológico $y$ de Estudios Superiores de Occidente, 2006 .

Gerchberg, A. "El concepto de la justicia inmanente. Estudio de un aspecto del desarrollo moral en niños y niñas entre seis y nueve años de edad en el área metropolitana". [Tesis de Licenciatura en Psicología]. Universidad de Costa Rica: 1994.

Marchesi, A. "El conocimiento social en los niños". Palacios, J.; Marchesi, A. y Carretero, M. (compiladores). Psicología evolutiva 2. Madrid. Alianza Editorial, 1997: 323-350.

Narayan, D. La voz de los pobres ¿Hay alguien que nos escuche? Banco Mundial: Ediciones Mundi-Prensa. 2000. En: $<$ http://www.fundacionpobreza.cl/fnp biblioteca.asp?Id_Carpeta $=350 \mathrm{yCamino}$ $=350 \% 7$ CPOBREZA $>$. [Consultado $15 \mathrm{de}$ mayo 2008].

Piaget, J. y Weil, A. (1951). "El desarrollo en el niño, la idea de patria y las relaciones con el extranjero". Delval, J. (compilador). Lecturas de psicología del niño. 2. El desarrollo cognitivo y afectivo del niño $y$ del adolescente. Madrid. Alianza Editorial, 1983: 325-342.

Rogoff, B. Aprendices del pensamiento. El desarrollo cognitivo en el contexto social. Barcelona: Ediciones Paidós, 1993.

Sánchez, G. "Teorías de niños y niñas sobre el castigo parental: un enfoque desde las emociones morales". [Tesis de Licenciatura en Psicología]. Universidad de Costa Rica. 2007.

Sandoval, C. La Carpio. La experiencia de segregación urbana y estigmatización. Instituto de Investigaciones Sociales, 2005. En: <http://ccp.ucr.ac.cr/noticias/ migraif/pdf/sandoval.pdf>. [Consultada el 17 de abril 2009]

Smith, V. "El proceso de construcción de la identidad étnica y su relación con la identidad". [Tesis de Licenciatura en Psicología]. Universidad de Costa Rica, 1995. 